\title{
An Approximation Algorithm for Optimal Consumption/Investment Problems*
}

\author{
Sanjiv Ranjan Das \\ Santa Clara University \\ Leavey School of Business \\ 500 El Camino Real \\ Santa Clara, CA 95053 \\ srdas@scu.edu
}

\author{
Rangarajan K. Sundaram \\ Stern School of Business \\ New York University \\ New York, NY 10012 \\ rsundara@stern.nyu.edu
}

June 20, 2001

${ }^{*}$ Comments Welcome. Special thanks to Luis Viceira and Raman Uppal for several sugestions. 


\title{
An Approximation Algorithm for Optimal Consumption/Investment Problems
}

\begin{abstract}
This article develops a simple approach to solving continuous-time portfolio choice problems. Portfolio problems for which no closed-form solutions are available may be handled by this technique, which substitutes the numerical solution of partial differential equations with a non-linear numerical algorithm approximating the solution. This paper complements the wide literature in economics on the solution of dynamic problems in discrete time. The algorithm is parismonious, and is illustrated by solving two examples, one, the standard Merton problem, and two, a jump-diffusion problem.
\end{abstract}




\section{Introduction}

The problem of optimal consumption and portfolio choice is one with a long history. Originally formulated in continuous time by Merton [27] [28], the problem has been extended substantially and several solution approaches have been developed. Barring the simplest problems, analytical solutions are difficult to come by. This paper provides a simple numerical approach to solving the optimal control problem using value function approximation.

For simpler problems, as in the original Merton formulation, closed-form solutions are achieved. The papers by Lehoczky, Sethi and Shreve [23], Karatzas, Lehoczky, Sethi and Shreve [20], Jacka [18], and Ocone and Karatzas [29] deal with explicit solutions, using the Bellman equation approach. The martingale approach of Cox and Huang [8] is also a well established one now, and has been extended to incomplete markets by He and Pearson [16] [17], Karatzas, Lehoczky, Shreve and $\mathrm{Xu}$ [21] and Cvitanic and Karatzas [9]. These problems are often further complicated by the choice of non-additive utility functions (see Duffie and Epstein [12], and more recently Dumas, Uppal and Wang [14]). Other complications arise when transactions costs are included in the analysis, as in Constantinides [7], Davis and Norman [10], and Dumas and Luciano [13].

In all these settings, simple versions admit either closed-form solutions or problems that are solved by applying simple numerical procedures. For example, in the case of the Bellman approach, if the number of state variables is low, the partial differential equation of optimality may be solved using finite-differencing methods. If the objective function is simple, then easily applied recursive methods may be used, as in Bertsimas, Kogan and Lo [1] where replication in incomplete markets is undertaken for a quadratic loss function, and the resulting system is quite tractable in low dimension. Bossaerts [2] examines a similar problem in an American option setting.

There are many approximation methods for the solution of these problems (see the review ar-

ticle by Taylor and Uhlig [32]). A wide variety of numerical approaches is applied such as iterating on the value function (Christiano [6]), quadrature methods (Tauchen [30] [31]), linear-quadratic approximations for the controls (Kydland and Prescott [22]), and parameterizations of the value 
function (Marcet [25], DeHaan and Marcet [11]). In this paper, we develop an analog of the parameterization approach in continuous-time, and demonstrate its application to a fairly general problem in continuous-time, that of a system driven by a mixed jump-diffusion stochastic process. More recent examples of approximation methods in the Finance literature include Campbell and Viceira [4], [5], Viceira [33], Brandt, Goyal and Santa-Clara [3], and Longstaff [24].

In continuous-time, solving the Bellman equation is more art than science. The usual approach involves making a clever guess as to the form of the value function, obtaining the optimal controls, and then verifying the solution after solving the Bellman PDE subject to the guess. Solutions have been obtained for some well-known and familiar utility functions, but whenever the number of state variables grows, or the stochastic processes chosen are not of the common geometric Brownian motion form, we are usually reliant on numerical schemes. This paper develops value function approximation as a method of extending the Bellman approach in a tractable way.

The basic idea is as follows. The optimal consumption-investment problem is set up as a Bellman control problem in the usual way. The first-order conditions provide the functional equations for the optimal controls, subject to solving for the value function. Rather than attempt to solve for the value function in closed form, we posit a very general polynomial form for the value function. Thus the value function is described as a general function of a finite parameter set, denoted $\theta$. Substituting this functional guess into the first-order conditions gives us the optimal controls as a function of $\theta$. These optimal controls are then plugged back into the Bellman equation which should hold for all possible outcomes of the state variables. This will only be true when the guess for the value function coincides with the true value function, and for complex problems, this is unlikely. However, if the approximation to the true value function is a good one, then the distance between the approximate value function and the exact one should be small over all points in the state space. Thus, our solution comprises of minimizing a "distance function" between exact and approximate value functions, by means of finding the best-fit parameter set $\theta$, subject to exactly satisfying the first-order conditions.

The algorithm works well, and we provide examples of its implementation in the paper. This 
approach has several benefits. First, it may be used to handle higher-dimensional problems, as well as problems with complex utility functions and stochastic processes. As an example, we solve a jump-diffusion model in the paper. Second, any standard minimizer routine may be applied for computational purposes making the problem computationally inexpensive. In fact, the illustrations in the paper use nothing more sophisticated than the optimizer in the Excel spreadsheet. Third, very general polynomial functions may be used to guess the value function. Thus, one may get arbitrarily close to the true value function for the problem. Finally, examination of the numerical solution provides hints as to the form of the true value function, which may lead to an explicit analytical solution.

In the following sections, we provide the problem set up, and the formal presentation of the solution method. Numerical examples are also provided with appropriate discussion.

\section{Stochastic Processes}

Investors face a state space that is characterized by an infinite trading interval $T=[0, \infty)$. The uncertainty in the portfolio choice set emanates from a set of diffusion processes and Poisson jump processes, with probability spaces $\left(\Omega^{Z}, F^{Z}, Q^{Z}\right)$ and $\left(\Omega^{N}, F^{N}, Q^{N}\right)$ respectively. $\mathbf{Z}_{\mathbf{t}} \in \mathbf{R}^{\mathbf{n}}$ represents a vector of Wiener processes defined on $\left(\Omega^{Z}, F^{Z}, Q^{Z}\right)$ and $\mathbf{N}_{\mathbf{t}} \in \mathbf{R}^{\mathbf{m}}$ represents a vector of orthogonal jump processes defined on $\left(\Omega^{N}, F^{N}, Q^{N}\right)$, where $t \in T$ and $m, n>0$. Each jump process is described by a sequence of random times $T^{(i)} \in T$ such that $N_{t, i}=\sum 1_{\left\{t \geq T^{(i)}\right\}}$. The Poisson jump arrival intensities are denoted $\lambda_{i}, i=1 \ldots m$. We allow for the jump intensities to vary stochastically on $T$ as well as functions of $\left\{\mathbf{Z}_{\mathbf{t}}, \mathbf{N}_{\mathbf{t}}\right\}$.

We also allow for $K$ state variables $\mathbf{x}_{\mathbf{t}} \in \mathbf{R}^{\mathbf{K}}$ which also evolve on the same jump-diffusion probability spaces defined above. These processes are defined as follows:

$$
d \mathbf{x}(\mathbf{t})=\alpha_{\mathbf{x}}(\mathbf{x}, \mathbf{t}) \mathbf{d} \mathbf{t}+\sigma_{\mathbf{x}}(\mathbf{x}, \mathbf{t}) \mathbf{d Z}(\mathbf{t})+\mathbf{J}_{\mathbf{x}}(\mathbf{x}, \mathbf{t}) \mathbf{d} \mathbf{N}(\lambda, \mathbf{t})
$$

where $\alpha_{\mathbf{x}}(\mathbf{x}, \mathbf{t}) \in \mathbf{R}^{\mathbf{K}}, \sigma_{\mathbf{x}}(\mathbf{x}, \mathbf{t}) \in \mathbf{R}^{\mathbf{K} \times \mathbf{n}}, \mathbf{J}_{\mathbf{x}}(\mathbf{x}, \mathbf{t}) \in \mathbf{R}^{\mathbf{K} \times \mathbf{m}}$. 
The economy offers a set of $L+1$ traded assets, whose values evolve stochastically on $T$. These assets comprise $L$ risky assets $(\mathbf{S})$ indexed $l=1 \ldots L$, and a riskless asset $(B)$ which earns a constant return $r$. Hence the stochastic process for this asset is

$$
d B(t)=r B(t) d t
$$

The remaining assets earn a random rate of return and obey the following SDE:

$$
\frac{d \mathbf{S}(\mathbf{t})}{\mathbf{S}(\mathbf{t})}=\alpha(\mathbf{S}, \mathbf{x}, \mathbf{t}) \mathbf{d} \mathbf{t}+\sigma(\mathbf{S}, \mathbf{x}, \mathbf{t}) \mathbf{d} \mathbf{Z}+\mathbf{J}(\mathbf{S}, \mathbf{x}, \mathbf{t}) \mathbf{d} \mathbf{N}(\lambda, \mathbf{t})
$$

where $\frac{d \mathbf{S}(\mathbf{t})}{\mathbf{S}(\mathbf{t})}, \alpha(\mathbf{S}, \mathbf{t}) \in \mathbf{R}^{\mathbf{L}}, \sigma(\mathbf{S}, \mathbf{t}) \in \mathbf{R}^{\mathbf{L} \times \mathbf{n}}$ and $\mathbf{J}(\mathbf{S}, \mathbf{t}) \in \mathbf{R}^{\mathbf{L} \times \mathbf{m}}$.

\section{Optimal Portfolio Choice}

The investor seeks to implement a consumption $\left(c_{t}\right)$ and portfolio plan $\left(\mathbf{w}_{\mathbf{t}}\right)$ so as to maximize his lifetime utility. The utility of consumption is given by the usual class of Von-Neumann and Morgenstern utility functions, which we denote $U\left(c_{t}\right)$, satisfying the usual requirements of concavity, and other technical regularity conditions.

The portfolio plan of the investor is a choice of asset weights $w_{l}, l=1 \ldots L$ such that the amount invested in the riskless asset is $w_{0}=1-\mathbf{w}^{\prime} 1$. At any time $t$, the investor chooses how much of his current wealth $W_{t}$ to consume, and invests the balance in the riskless and risky assets. Thus, the stochastic process for wealth taking into account investment and consumption is as follows (see Merton [26] for details):

$$
d W=\left\{W\left[\mathbf{w}^{\prime}(\alpha-\mathbf{r} \mathbf{1})+\mathbf{r}\right]-c\right\} d t+W \mathbf{w}^{\prime} \sigma \mathbf{d} \mathbf{Z}+\mathbf{W w}^{\prime} \mathbf{J d} \mathbf{N}
$$

At the initial time $t=0$, we are interested in solving for the investor's optimal consumption investment program, so as to undertake the following maximization:

$$
\max _{\{c, \mathbf{w}\}} E_{0}\left\{\int_{0}^{\infty} e^{-\rho s} U\left(c_{s}\right) d s\right\}
$$


where $\rho$ is the investor's time preference parameter, a scalar constant. The optimized function at any time $t$ is denoted as the value function, defined recursively as a function of state variables $\{W, \mathbf{x}\}$

$$
V(W, \mathbf{x} ; \mathbf{t})=\max _{\mathbf{c}_{\mathbf{t}}, \mathbf{w}_{\mathbf{t}}} \mathbf{E}_{\mathbf{t}}\left\{\mathbf{U}\left(\mathbf{c}_{\mathbf{t}}\right)+\mathbf{V}(\mathbf{W}, \mathbf{x} ; \mathbf{t}+\mathbf{d t})\right\} .
$$

Using the method of stochastic dynamic programming (see Merton [26]), we arrive at the Bellman equation of optimality

$$
0=\max _{\{c, \mathbf{w}\}} H(W, \mathbf{x})
$$

which in full detailed form is:

$$
\begin{aligned}
0= & \max _{\{c, \mathbf{w}\}}\left\{U(c)-\rho V+V_{W} W\left[\mathbf{w}^{\prime}(\alpha-\mathbf{r} \mathbf{1})+\mathbf{r}\right]-c \frac{\partial V}{\partial W}\right. \\
& +\frac{1}{2} V_{W W} W^{2} \mathbf{w}^{\prime} \sigma \sigma^{\prime} \mathbf{w}+\sum_{\mathbf{j}=\mathbf{1}}^{\mathbf{m}} \mathbf{E}\left(\lambda_{\mathbf{j}}\left[\mathbf{V}\left(\mathbf{W}+\mathbf{w}^{\prime} \mathbf{J}_{\mathbf{j}} \mathbf{W}, \mathbf{x}\right)-\mathbf{V}(\mathbf{W}, \mathbf{x})\right]\right) \\
& +V_{\mathbf{x}}^{\prime} \alpha_{\mathbf{x}}+\frac{\mathbf{1}}{\mathbf{2}} \sigma_{\mathbf{x}}^{\prime} \mathbf{V}_{\mathbf{x} \mathbf{x}} \sigma_{\mathbf{x}}+\mathbf{V}_{\mathbf{W} \mathbf{x}}^{\prime} \sigma_{\mathbf{x}} \sigma^{\prime} \mathbf{w} \mathbf{W} \\
& \left.+\sum_{j=1}^{m} E\left(\lambda_{j}\left[V\left(W, \mathbf{x}+\mathbf{J}_{\mathbf{x}}\right)-\mathbf{V}(\mathbf{W}, \mathbf{x})\right]\right)\right\}
\end{aligned}
$$

where $\mathbf{J}_{\mathbf{j}} \in \mathbf{R}^{\mathbf{1}}$ is the $j$ th column of matrix $\mathbf{J} \in \mathbf{R}^{\mathbf{L} \times \mathbf{m}}$. Subscripts denote partial derivatives, i.e. $V_{w}=\frac{\partial V}{\partial W}, V_{W W}=\frac{\partial^{2} V}{\partial W^{2}}$. Likewise, $V_{\mathbf{x}}=\frac{\partial V}{\partial \mathbf{x}} \in R^{K}, V_{\mathbf{x x}}=\frac{\partial^{2} V}{\partial \mathbf{x} \partial \mathbf{x}^{\prime}} \in R^{K \times K}$ and $V_{W \mathbf{x}}=\frac{\partial V}{\partial W \partial \mathbf{x}^{\prime}} \in$ $R^{K}$. The solution method entails taking the first-order derivatives from the equation of optimality to arrive at the optimal controls $\{c, \mathbf{w}\}$, as functions of $V(W, \mathbf{x})$. Then, the substitution of these values into the Bellman equation provides a second order $(K+1)$-dimensional partial differential equation in $(W, \mathbf{x})$ which must be solved subject to suitably imposed boundary conditions.

The first-order condition for consumption,

$$
U^{\prime}(c)=V_{W}
$$

implies the optimal consumption rule: $c^{*}=I\left(\frac{\partial V}{\partial W}\right), I=\left[U^{\prime}\right]^{-1}, U^{\prime}=\frac{\partial U}{\partial c}$. Taking the first-order condition for optimal portfolio weights $\mathbf{w}^{*}$, we get the $(L \times 1)$ equation system

$$
0=V_{W}(\alpha-\mathbf{r} \mathbf{1}) W+V_{W W} \sigma \sigma^{\prime} \mathbf{w} \mathbf{W}^{\mathbf{2}}
$$




$$
\begin{aligned}
& +\sum_{j=1}^{m} E\left(\lambda_{j}\left[\frac{\partial}{\partial \mathbf{w}} V\left(W+\mathbf{w}^{\prime} \mathbf{J}_{\mathbf{j}} \mathbf{W}\right)-\mathbf{V}(\mathbf{W}) \mathbf{1}\right]\right) \\
& +\sigma \sigma_{\mathbf{x}}^{\prime} \mathbf{V}_{\mathbf{W} \mathbf{x}} \mathbf{W}
\end{aligned}
$$

where $\frac{\partial}{\partial \mathbf{w}} V\left(W+\mathbf{w}^{\prime} \mathbf{J}_{\mathbf{j}} \mathbf{W}\right) \in \mathbf{R}^{\mathbf{L}}$.

\section{The Approximation Algorithm}

Exact solution of the problem in Section 3 is usually hard to achieve, except in the simplest of cases. The problem lies in the fact that the optimal controls are complicated functions of the state variables, and value function, which itself is the solution to a high-dimensional differential equation. The usual approach is to guess a functional form for the value function and then verify whether it satisfies the optimality conditions of the problem. Apart from a few well-known cases, this approach has proven rather fruitless. Alternatively, one could attempt to solve the differential equation using numerical methods such as finite-differencing, but achieving a stable numerical scheme with many state variables has proven to be a daunting task.

Here, we suggest an alternative approach which bypasses these problems. The idea is to posit a polynomial function of the state variables as an approximation to the value function. We choose a $\theta$-parameterized function $V(\theta) \equiv V(W, \mathbf{x} ; \theta)$, where $\theta \in R^{P+1}$ is a set of parameters $\left\{v_{0}, v_{1} \ldots v_{P}\right\}$ which define the value function. If we are able to find the "best" possible value function $V^{*}(\theta)$, then we have automatically obtained the solution to the problem, since the controls $\{c, \mathbf{w}\}$ derive immediately. The exact solution will satisfy

$$
0=H\left(W, \mathbf{x} ; \mathbf{V}^{*}\right)
$$

subject to satisfying the constraints from the first-order conditions, in equations (9) and (10). If we are not able to find the optimal value function, we can find the best $V$ in a set of value functions $\{V(\theta)\}$ which may be chosen arbitrarily. To do this we compute the following optimization program:

$$
\min _{\{V(\theta)\}}\left\{\min _{\theta} \sum_{u \in U} f(H[W(u), \mathbf{x}(u) ; V(W, \mathbf{x} ; \theta)])\right\}
$$


subject to

$$
\begin{array}{rlrl}
w & =w^{*}[V(W(u), \mathbf{x}(u) ; \theta)], & \forall u \\
c=c^{*}[V(W(u), \mathbf{x}(u) ; \theta)], & \forall u .
\end{array}
$$

Here, $U$ represents a discrete set of choices of state variables, i.e. a chosen state-space for the problem. These values $u=\{W(u), \mathbf{x}(u)\}$ may be chosen to reflect the decision-makers envisaged outcomes of the state variables. The function $f($.$) is the fitting function and may$ be chosen from a range of popular options. For example, a least-squares approach would set $f(H)=H^{2}$. Alternatively, a probability weighted function such as $f(H)=H^{2} \times \operatorname{prob}(u)$ may be used. A simple absolute valued function $f(H)=|H|$ is also possible. Optimization is undertaken by choosing a specific $V(\theta)$ and then optimizing. Searching over the set $\{V(\theta)\}$ will produce the best value function.

This approach has certain advantages. First, it does not require the solution of a highdimensional differential equation. Second, the complexity of form of the value functional $V(\theta)$ does not impact substantially the computational requirements of the algorithm. The number of points in the state space $U$ does however increase the number of constraints to be satisfied in a linear way. But this was not found to be numerically difficult, and in fact, implementation with a spreadsheet optimizer works almost all the time.

The following section provides illustrations of the model implementation.

\section{Illustrative Examples}

\subsection{A simple implementation example}

Consider the following simple problem. We begin with a single asset setting, where the asset follows a geometric Brownian motion.

$$
d S=\alpha S d t+\sigma S d Z
$$


The notation follows from the previous section. Assume a power utility function over consumption where $U(c)=\frac{1}{\eta} c^{\eta}$. Analogous to equation (8) the Bellman equation of optimality will be:

$$
0=\max _{c, w}\left\{U(c)-\rho V+V_{W} W[w R+r]-V_{W} c+\frac{1}{2} V_{W W} w^{2} W^{2} \sigma^{2}\right\}
$$

where $R=\alpha-r$ defines the equity premium/excess return. The first-order condition for consumption gives $U^{\prime}(c)=V_{W}$ which implies that the optimal consumption is $c^{*}=\left(V_{W}\right)^{\frac{1}{\eta-1}}$. Likewise, the first-order condition for the portfolio weights gives the optimal investment in the risky asset via the equation $V_{W} R+V_{W W} w W \sigma^{2}=0$, implying that $w^{*}=-\frac{R}{\sigma^{2}} \frac{V_{W}}{W V_{W W}}$. As is well known from Merton [26], the solution to this problem provides a value function of the form $V(W)=A \frac{W^{\eta}}{\eta}$, which implies that the optimal weights are $w^{*}=\frac{R}{\sigma^{2}} \frac{1}{(1-\eta)}$.

Now suppose we did not know the value function form in advance, and made a guess as to its form by choosing a somewhat more general function. Let $V(W)=V_{0}+V_{1} W^{V_{2}}$ where $\left(V_{0}, V_{1}, V_{2}\right)$ are unknown scalar constants. ${ }^{1}$ Our approach then entails substituting this posited value function into the Bellman equation and solving for the best fit values of $\left(V_{0}, V_{1}, V_{2}\right)$ over the state space, which comprises a range of values of $W$.

Let the vector of $N$ values of $W$ be indexed by $i$, such that we have $W_{i}, i=1 \ldots N$. This then defines a vector of values of the Bellman equation. Denote the optimized Bellman equation vector as $M_{i}, i=1 \ldots N$. Thus, we have

$$
M_{i}=U\left(c_{i}^{*}\right)-\rho V+V_{W} W_{i}\left[w_{i}^{*} R+r\right]-V_{W} c_{i}^{*}+\frac{1}{2} V_{W W} w_{i}^{* 2} W_{i}^{2} \sigma^{2}, \quad \forall W_{i} .
$$

Note here that the equation contains the optimized values $\left(c_{i}^{*}, w_{i}^{*}\right)$ from the first-order conditions. Also, the derivatives $V_{W}$ and $V_{W W}$ are functions of $W_{i}$ but the notation in the form $V_{W}\left(W_{i}\right)$ has been suppressed for expositional reasons.

Assume any unconditional distribution for $W_{i}$. Denote the probability of $W_{i}$ as $f\left(W_{i}\right)$, and $\sum_{i=1}^{N} f\left(W_{i}\right)=1$. For the optimal value function, it must be that $M_{i}=0, \forall i$. However, since we are guessing the value function, and may not detect its exact form, the best that is possible is

\footnotetext{
${ }^{1}$ This approximation varies from other approaches using simple ploynomial forms, such as in the Galerkin method (see Judd [19], pg 373).
} 
to choose $\left(V_{0}, V_{1}, V_{2}\right)$ so as to make the values of $\left|M_{i}\right|, \forall i$ as small as possible. This suggests a range of objective functions of which an example is provided below.

$$
\min _{\left\{V_{0}, V_{1}, V_{2}\right\}} \sum_{i=1}^{N}\left\{M_{i}^{2} f\left(W_{i}\right)\right\}
$$

Under the assumption of equally weighted occurences of $W_{i}$, this is simply the least squares method.

We now explore the solution in more detail. First, we can compute the analytical derivatives of the value function. We get

$$
\begin{aligned}
V_{W} & =V_{1} V_{2} W_{i}^{V_{2}-1} \\
V_{W W} & =V_{1} V_{2}\left(V_{2}-1\right) W_{i}^{V_{2}-2} \\
c_{i}^{*} & =\left[V_{1} V_{2} W_{i}^{V_{2}-1}\right]^{\frac{1}{\eta-1}} \\
w_{i}^{*} & =\frac{R}{\sigma^{2}} \frac{1}{\left(1-V_{2}\right)} .
\end{aligned}
$$

The set of Bellman equations becomes (for every $i$ )

$$
\begin{aligned}
M_{i}= & \frac{1}{\eta}\left[V_{1} V_{2} W_{i}^{V_{2}-1}\right]^{\frac{\eta}{\eta-1}}-\rho\left[V_{0}+V_{1} W^{V_{2}}\right] \\
& +V_{1} V_{2} W_{i}^{V_{2}-1}\left[\frac{R}{\sigma^{2}} \frac{1}{\left(1-V_{2}\right)}+r\right] \\
& -V_{1} V_{2} W_{i}^{V_{2}-1}\left[V_{1} V_{2} W_{i}^{V_{2}-1}\right]^{\frac{1}{\eta-1}} \\
& +\frac{1}{2} V_{1} V_{2}\left(V_{2}-1\right) W_{i}^{V_{2}-2}\left[\frac{R}{\sigma^{2}} \frac{1}{\left(1-V_{2}\right)}\right]^{2} W_{i}^{2} \sigma^{2}, \quad \forall i .
\end{aligned}
$$

The minimization problem may be simply stated as $\min _{\left\{V_{0}, V_{1}, V_{2}\right\}} \sum_{i=1}^{N} M_{i}^{2}$. Since we impose $V(W=0)=0$, we get that $V_{0}=0$. Further, it may easily be checked that the numerical minimization in fact leads to the solution $V_{1}>0, V_{2}=\eta$. This matches exactly the solutions in Merton [28]. Hence, the technique provides the known solution. We now proceed to a numerical illustration of a more complex model. 


\subsection{An example with jump processes}

We extend the process driving the risky asset to including jumps with stochastic intensity. Thus,

$$
\begin{aligned}
& d S=\alpha S d t+\sigma S d Z+J S d N(\lambda) \\
& d \lambda=k(\theta-\lambda) d t+\delta \sqrt{\lambda} d Y .
\end{aligned}
$$

Thus, the jump intensity $\lambda$ follows a mean-reverting square-root diffusion, and we assume that $d Z, d Y$ are orthogonal diffusions. The value function is now extended to cover the new state variable $\lambda$ in addition to wealth, so that we write it as $V(W, \lambda)$. From (8) we get the Bellman equation:

$$
\begin{aligned}
0= & \max _{c, w}\left\{U(c)-\rho V(W, \lambda)+V_{W} W[w R+r]-V_{W} c+\frac{1}{2} V_{W W} w^{2} W^{2} \sigma^{2}\right. \\
& \left.+V_{\lambda} k(\theta-\lambda)+\frac{1}{2} V_{\lambda \lambda} \delta^{2} \lambda+\lambda E[V(W+w W J, \lambda)-V(W, \lambda)]\right\}
\end{aligned}
$$

We guess the following functional form for the value function

$$
V(W, \lambda)=V_{0}+V_{1} W^{V_{2}}+V_{3} \lambda^{V_{4}}+V_{5} W \lambda
$$

which yields the following terms

$$
\begin{aligned}
V_{W} & =V_{1} V_{2} W^{V_{2}-1}+V_{5} \lambda \\
V_{W W} & =V_{1} V_{2}\left(V_{2}-1\right) W^{V_{2}-2} \\
V_{\lambda} & =V_{3} V_{4} \lambda^{V_{4}-1}+V_{5} W \\
V_{\lambda \lambda} & =V_{3} V_{4}\left(V_{4}-1\right) \lambda^{V_{4}-2}
\end{aligned}
$$

From the first-order condition for consumption, we obtain the optimal value of $c$

$$
c^{*}=\left[V_{1} V_{2} W^{V_{2}-1}+V_{5} \lambda\right]^{\frac{1}{\eta-1}} .
$$

The first-order condition for the risky asset weights is

$$
V_{W} W R+V_{W W} w \sigma^{2} W^{2}+\lambda E\left[\frac{\partial}{\partial w} V[W+w W J]\right]=0 .
$$


Thus, $w^{*}$ is implicitly defined as the solution to the above equation. The last term requires

$$
V[W+w W J]=V_{0}+V_{1} W^{V_{2}}(1+w J)^{V_{2}}+V_{3} \lambda^{V_{4}}+V_{5} W(1+w J) \lambda,
$$

which provides

$$
\frac{\partial}{\partial w} V[W+w W J]=V_{1} V_{2} W^{V_{2}}(1+w J)^{V_{2}-1} J+V_{5} W \lambda J .
$$

For the purposes of this example we assume a binary form for the jump, i.e

$$
J=\left\{\begin{array}{cc}
+j, & \mathrm{w} / \text { prob } \frac{1}{2} \\
-j, & \mathrm{w} / \operatorname{prob} \frac{1}{2}
\end{array}\right.
$$

which leads to

$$
\begin{aligned}
E[V(W, \lambda)]= & V_{0}+V_{1} W^{V_{2}}+V_{3} \lambda^{V_{4}}+V_{5} W \lambda \\
E\{V[W+w W J, \lambda]\}= & V_{0}+V_{1} W^{V_{2}} \times \frac{1}{2}\left[(1+w j)^{V_{2}}+(1-w j)^{V_{2}}\right] \\
& +V_{3} \lambda^{V_{4}}+V_{5} W \lambda \\
E\left\{\frac{\partial}{\partial w} V[W+w W J, \lambda]\right\}= & \frac{1}{2}\left[V_{1} V_{2} W^{V_{2}}(1+w j)^{V_{2}-1} j\right. \\
& \left.-V_{1} V_{2} W^{V_{2}}(1-w j)^{V_{2}-1} j\right] .
\end{aligned}
$$

The first-order condition for portfolio weights may now be written as

$$
\begin{aligned}
0= & {\left[V_{1} V_{2} W^{V_{2}-1}+V_{5} \lambda\right] W R+\left[V_{1} V_{2}\left(V_{2}-1\right) W_{i}^{V_{2}-2}\right] w \sigma^{2} W^{2} } \\
& +\lambda \frac{1}{2}\left[V_{1} V_{2} W^{V_{2}}(1+w j)^{V_{2}-1} j-V_{1} V_{2} W^{V_{2}}(1-w j)^{V_{2}-1} j\right] .
\end{aligned}
$$

The vector of Bellman equations is now written as ( $i$ now indexes the joint space over state variables $W, \lambda)$ :

$$
\begin{aligned}
M_{i}= & U\left(c_{i}^{*}\right)-\rho V\left(W_{i}, \lambda_{i}\right) \\
& +\left(V_{1} V_{2} W_{i}^{V_{2}-1}+V_{5} \lambda\right) W_{i}\left[w_{i}^{*} R+r\right]-\left(V_{1} V_{2} W_{i}^{V_{2}-1}+V_{5} \lambda\right) c_{i}^{*} \\
& +\frac{1}{2}\left(V_{1} V_{2}\left(V_{2}-1\right) W_{i}^{V_{2}-2}\right) w_{i}^{* 2} W_{i}^{2} \sigma^{2} \\
& +\left(V_{3} V_{4} \lambda_{i}^{V_{4}-1}+V_{5} W_{i}\right) k\left(\theta-\lambda_{i}\right)
\end{aligned}
$$




$$
\begin{aligned}
& +\frac{1}{2}\left(V_{3} V_{4}\left(V_{4}-1\right) \lambda_{i}^{V_{4}-2}\right) \delta^{2} \lambda_{i} \\
& +\lambda_{i}\left\{V_{0}+V_{1} W_{i}^{V_{2}} \times \frac{1}{2}\left[\left(1+w_{i}^{*} j\right)^{V_{2}}+\left(1-w_{i}^{*} j\right)^{V_{2}}\right]+V_{3} \lambda_{i}^{V_{4}}+V_{5} W_{i} \lambda_{i}\right\} \\
& -\lambda_{i}\left\{V_{0}+V_{1} W_{i}^{V_{2}}+V_{3} \lambda_{i}^{V_{4}}+V_{5} W_{i} \lambda_{i}\right\} .
\end{aligned}
$$

Since the first-order condition for portfolio weights $w$ is an implicit equation, we solve the following optimization problem to obtain the values $\left(V_{0}, V_{1}, V_{2}, V_{3}, V_{4}, V_{5}\right)$ :

$$
\min _{\left\{V_{0}, V_{1}, V_{2}, V_{3}, V_{4}, V_{5}\right\}} \sum_{i=1}^{N} M_{i}^{2}
$$

This problem is solved numerically, and the results are provided below. Additionally, since the jump-diffusion based problem nests the pure-diffusion model, we can examine the features of the first solution from Section 5.1 as well.

\subsection{Numerical results}

The following parameters were chosen as a base case for the jump-diffusion model.

\begin{tabular}{lcc}
\hline \hline Parameter Description & Notation & Value \\
\hline \hline Relative risk aversion & $\eta$ & 0.5 \\
Mean return on risky asset & $\alpha$ & 0.07 \\
Riskless rate & $r$ & 0.03 \\
Subjective discount rate & $\rho$ & 0.03 \\
Volatility coefficient for risky asset & $\sigma$ & 0.3 \\
\hline Mean reversion for jump intensity $(\lambda)$ & $k$ & 0.5 \\
Mean level of $\lambda$ & $\theta$ & 7.5 \\
Volatility coefficient of $\lambda$ & $\delta$ & 5 \\
Jump amplitude & $j$ & 0.1 \\
\hline \hline
\end{tabular}

In order to solve the problem we need to establish a state space $\left(W_{i}, \lambda_{i}\right), \forall i$. We used a range of values of $W \in[0,10]$ and for jump intensity we assumed a two-state model where $\lambda \in\{5,10\}$, 
i.e. low and high jump states. The algorithm was implemented on an Excel spreadsheet, and converges in a few seconds. The optimal values $\left(V_{0}, V_{1}, V_{2}, V_{3}, V_{4}, V_{5}\right)$ are:

\begin{tabular}{cccccc}
\hline \hline \multicolumn{6}{c}{$\begin{array}{c}\text { Optimal Value Function Parameters } \\
\text { (Jump-diffusion model) }\end{array}$} \\
\hline \hline$V_{0}$ & $V_{1}$ & $V_{2}$ & $V_{3}$ & $V_{4}$ & $V_{5}$ \\
0 & 14.1290 & 0.4995 & -1.0231 & -0.0198 & -0.0021 \\
\hline \hline
\end{tabular}

The signs of the parameters are exactly as expected. Note that $V_{1}, V_{2}$ are greater than zero, since indirect utility is increasing in the level of wealth. Likewise $V_{3}, V_{4}, V_{5}$ are less than zero, since utility declines when jump risk increases. For the purposes of comparison, we switched off the jump process to reduce the problem to the pure-diffusion model. To do so, we set the parameters as follows: $(k, \theta, \delta, j, \lambda)$ to zero. In this setting, the value function is:

\begin{tabular}{ccccccc}
\hline \hline $\begin{array}{c}\text { Optimal Value Function Parameters } \\
\text { (Pure-diffusion model) }\end{array}$ \\
\hline \hline$V_{0}$ & $V_{1}$ & $V_{2}$ & $V_{3}$ & $V_{4}$ & $V_{5}$ \\
0 & 18.0889 & 0.5000 & 0 & 0 & 0 \\
\hline \hline
\end{tabular}

This solution corresponds exactly to that of the known solution in Merton [26]. Notice that the value of $V_{2}=\eta$ as required in theory. In the following table we present some of the qualitative results from the two models, and undertake a comparison of outcomes. 


\begin{tabular}{c|c|c|c|ccc|ccc}
\hline \hline \multicolumn{8}{c}{ Optimal Consumption and Investment Values } \\
\hline \hline & \multicolumn{3}{|c|}{ Pure-diffusion model } & \multicolumn{3}{c}{ Jump-diffusion model } \\
& \multicolumn{3}{|c}{$\lambda=0$} & \multicolumn{3}{c}{$\lambda=5$} & \multicolumn{3}{c}{$\lambda=10$} \\
\hline$W$ & $c^{*}$ & $w^{*}$ & $V(W, \lambda)$ & $c^{*}$ & $w^{*}$ & $V(W, \lambda)$ & $c^{*}$ & $w^{*}$ & $V(W, \lambda)$ \\
\hline 0.1 & 0.0012 & 0.8888 & 5.72 & 0.0020 & 0.5702 & 3.24 & 0.0020 & 0.4200 & 3.25 \\
0.9 & 0.0110 & 0.8888 & 17.16 & 0.0181 & 0.5697 & 12.16 & 0.0182 & 0.4192 & 12.17 \\
2.5 & 0.0306 & 0.8888 & 28.60 & 0.0505 & 0.5691 & 21.07 & 0.0507 & 0.4184 & 21.06 \\
4.5 & 0.0550 & 0.8888 & 38.37 & 0.0910 & 0.5687 & 28.67 & 0.0916 & 0.4177 & 28.64 \\
10.1 & 0.1235 & 0.8888 & 57.48 & 0.2052 & 0.5678 & 43.52 & 0.2071 & 0.4164 & 43.42 \\
\hline \hline
\end{tabular}

The table presents results from the pure-diffusion model, and the jump-diffusion model. For varying levels of the state variables $W$ and $\lambda$, we examine three values of interest: optimal consumption, investment in the risky asset, and the value function.

First, we note that as the level of wealth increases, so does the value function. Second, as jump intensity increases, investor utility decreases since additional risk is borne. The only exception occurs when wealth is at a very low level and the jump intensity increases from 5 to 10 . This may be on account of the fact that at low levels of wealth, additional jumps cannot harm the investor given a floor level of zero on wealth. Plus, at high levels of jump intensity, mean reversion will lower jump risk. Third, as wealth increases, the investor consumes more. Fourth, as jump risk increases the investor also consumes more, since investing becomes less attractive, and consumption from the future is shifted to the present. Fifth, as jump risk increases, the investor correspondingly invests less in the risky asset. Sixth, when there is no jump risk, the amount invested in the risky asset is independent of wealth, as is known from the Merton model. However, when jump risk exists, the hedging term in equation (23) comes into play, and the choice of risky assets is no longer independent of wealth level. Finally, jump risk has a greater effect on the investor decision when jump risk is small and increasing than when it is large and increasing. 


\section{Concluding Comments}

This paper develops a simple numerical approach to solving optimal consumption and investment problems when analytic solutions are not achievable. The Bellman problem is translated into an econometric one, where we minimize a parameterized objective function of the Bellman equation over the state space, based on a polynomial guess for the value function. The method is tractable, and converges quickly. The approach offers a means to numerically guessing the form of the value function which, if fortuitous, may lead to an analytic solution.

The algorithm may be extended to solving problems in other domains in finance, such as asset-liability management, optimal replication of derivative securities, equilibrium problems with incomplete markets and market micostructure games.

\section{References}

[1] BERTSIMAS, D., L. KOGAN, and A. LO (1997) "Pricing and Hedging Derivative Securities in Incomplete Markets: An $\in$ - Arbitrage Approach," working paper No. 6250, NBER.

[2] BOSSAERTS, P. (1989) "Simulation Estimators of Optimal Early Exercise," working paper, Carnegie Mellon University.

[3] BRANDT, M., A. GOYAL, and P. SANTA-CLARA (2000). "Dynamic Portfolio Choice with Simulation Methods," working paper, UCLA.

[4] CAMPBELL, J., and L. VICEIRA (1996) "Consumption and Portfolio Decisions when Expected returns are Time Varying," NBER working paper no. 5857.

[5] CAMPBELL, J., and L. VICEIRA (1996) "Who should buy long-term bonds?" unpublished paper, Harvard University. 
[6] CHRISTIANO, L.J. (1990) "Solving the Stochastic Growth Model by Linear-Quadratic Approximation and by Value-Function Iteration," Journal of Business and Economic Statistics, v8(1), 23-26.

[7] CONSTANTINIDES, G. (1986) "Capital Market Equilibrium with Transactions Costs," Journal of Political Economy, v94, 842-862.

[8] COX, D., and C-F. HUANG (1989) "Optimal Consumption and Portfolio Policies when Asset Prices follow a Diffusion Process," Journal of Economic Theory, v49, 33-83.

[9] CVITANIC, J., and I. KARATZAS (1991) "Convex Duality in Constrained Portfolio Optimization," working paper, Columbia University.

[10] DAVIS, M., and A. NORMAN (1990) "Portfolio Selection with Transaction Costs," Mathematics of Operations Research, v15, 676-713.

[11] DEN HAAN, W.J., and A. MARCET (1990) "Solving the Stochastic Growth Model by Parameterizing Expectations," Journal of Business and Economic Statistics, v8(1), 31-34.

[12] DUFFIE, D., and L. EPSTEIN (1992) "Stochastic Differential Utility," Econometrica, v60, 353-394.

[13] DUMAS, B., and E. LUCIANO (1989) "An Exact Solution to a Dynamic Portfolio Choice Problem with Transations Costs," working paper, Wharton School, University of Pennsylvania.

[14] DUMAS, B., R. UPPAL and T. WANG (1997) "Efficient Intertemporal Allocations with Recursive Utility," working paper, HEC School of Management, and University of British Columbia.

[15] FLEMING, W., and R. RISHEL (1975) Deterministic and Stochastic Optimal Control, Berlin: Springer-Verlag. 
[16] HE, H., and N. PEARSON. (1991) "Consumption and Portfolio Policies with Incomplete Markets: The Infinite-Dimensional Case," Journal of Economic Theory, v54, 259-305.

[17] HE, H., and N. PEARSON. (1991) "Consumption and Portfolio Policies with Incomplete Markets: The Finite-Dimensional Case," Mathematical Finance, v1, 1-10.

[18] JACKA, S. (1984) "Optimal Consumption of an Investment," Stochastics, v13, 45-60.

[19] Judd, K. (1998) "Numerical Methods in Economics," MIT Press, Cambridge, MA.

[20] KARATZAS, I., J. LEHOCZKY, S. SETHI and S. SHREVE (1986) "Explicit Solution of a General Consumption/Investment Problem," Mathematics of Operations Research, v11, 261-294.

[21] KARATZAS, I., J. LEHOCZKY, S. SHREVE and G-L. XU (1991) "Martingale and Duality Methods for Utility Maximization in Incomplete Markets," SIAM Journal of Control and Optimization, v29, 702-730.

[22] KYDLAND, F., and E. PRESCOTT (1982) "Time to Build and Aggregate Flucutations," Econometrica, v50, 1345-1370.

[23] LEHOCZKY, J., S. SETHI, and S. SHREVE (1983) "Optimal Consumption and Investment Policies allowing Consumption Constraints and Bankruptcy," Mathematics of Operations Research, v8, 613-636.

[24] LONGSTAFF, F (2000) "Optimal Portfolio Choice and the Valuation of Illiquid Securities," forthcoming Review of Financial Studies.

[25] MARCET, A. (1988) "Solving Non-Linear Models by Parameterizing Expectations," working paper, Graduate School of Industrial Administration, Carnegie-Mellon University.

[26] MERTON, R.C. (1992) "Continuous-Time Finance," Basil Blackwell, New York.

[27] MERTON, R.C. (1969) "Lifetime Portfolio Selection under Uncertainty: The ContinuousTime Case," Review of Economics and Statistics, v51, 247-257. 
[28] MERTON, R.C. (1971) "Optimum Consumption and Portfolio Rules in a Continuous Time Model," Journal of Economic Theory, v3, 373-413.

[29] OCONE, D., and I. KARATZAS (1991) "A Generalized Clark Representation Formula, with Application to Optimal Portfolios," Stochastics and Stochastics Reports, v34, 187-220.

[30] TAUCHEN, G. (1987) "Quadrature-Based Methods for Obtaining Approximate Solutions to Non-linear Asset Pricing Models," working paper, Duke University, Dept of Economics.

[31] TAUCHEN, G. (1990) "Solving the Stochastic Growth Model by using Quadrature Methods and Value-Function Iterations," Journal of Business and Economic Statistics, v8(1), 49-51.

[32] TAYLOR, J.B., and H. UHLIG (1990) "Solving Non-Linear Stochastic Growth Models: A Comparison of Alternative Solution Methods," Journal of Business and Economic Statistics, v8(1), 1-17.

[33] VICEIRA, L. (1997) "Optimal Portfolio Choice for Long-horizon Investors with Nontradeable Income," unpublished paper, Harvard University. 